\title{
Development and Application of 3D Printing and Its Materials
}

\author{
Haocheng Du*, Shaoqian Chen \\ College of Science \& Technology, Ningbo University, Cixi 315300, Zhejiang Province, China. E-mail: \\ 1140752582@qq.com
}

\begin{abstract}
With the improvement of 3D printing technology, it has gradually gained popularity in China, and it has become famous for its wide variety of printing biological materials. This article will analyze and research the application and development of 3D printing technology in chemical materials. Explain the market demand for 3D printing technology, as well as the problems existing in the development of domestic 3D printing technology, and predict its development prospects from the development field.
\end{abstract}

Keywords: 3D Printing; Chemical Materials; Market Prospects; Development Routes; Application Areas

\section{Overview of $3 D$ printing industry}

The so-called "3D printing” is the general term for a series of rapid prototyping technology, which belongs to a kind of manufacturing technology. It is quite different from the traditional printing method. The principle is laminated manufacturing, which is formed by a rapid prototyping machine in the XY plane through scanning. For the cross-sectional shape of the thin layer of the workpiece, the thickness is superimposed in the $\mathrm{Z}$ direction, and the final superimposition forms a three-dimensional product. The characteristic is that it can create objects of almost any shape ${ }^{[1]}$.

The process is roughly as follows: first build a three-dimensional model through CAD or modeling software, and then scan the cross-section of the built models layer by layer. The printer reads the cross-sectional information in the file and uses liquid, powder or sheet materials to combine them. The cross-sections are printed layer by layer, and then the cross-sections of each layer are glued together in various ways to create a solid body. It can process and manufac- ture samples of more complicated original embryos, while greatly improving the speed and efficiency of processing and manufacturing, which plays a great saving effect on reducing related input costs. Some large, medium and small domestic manufacturing companies can often see the application of 3D printing technology.

\section{Application of chemical materials in $3 D$ printing}

Chemical materials have become the main materials in China's industry, manufacturing, and construction industries, and their importance has been at the forefront of the country, occupying a large share. The application cost of 3D printing materials is relatively high, but its efficiency and scale have obvious advantages. In particular, it will have an impact on the related chemical market, raw material processing and other related chain industries $^{[2]}$.

\subsection{Classification of 3D printing chemical materials}

\subsubsection{Polymer materials for 3D printing}

Copyright (C) 2020 Haocheng Du and Shaoqian Chen

doi: 10.18282/ims.v3i2.331

This is an open-access article distributed under the terms of the Creative Commons Attribution Non-Commercial License

(http://creativecommons.org/licenses/by-nc/4.0/), which permits unrestricted non-commercial use, distribution, and reproduction in any medium, provided the original work is properly cited. 
Polymer materials for 3D printing mainly include photosensitive resins, thermoplastics, and hydrogels. Photosensitive resin is one of the earliest materials used in $3 \mathrm{D}$ printing. It is suitable for light curing and forming. The main component is a small molecule resin that can undergo polymerization. It is added with photoinitiators, polymerization inhibitors, leveling agents and other additives. Under specific light (usually ultraviolet light), polymerization occurs to achieve curing.

Thermoplastic polymers are one of the most common 3D printing materials. Common thermoplastic polymers for 3D printing include acrylonitrile-butadiene-styrene (ABS), polylactic acid (PLA), nylon (PA), and polycarbonate. (PC), polystyrene (PS), polycaprolactone (PCL), polyphenylsulfone (PPSF), thermoplastic polyurethane (TPU), polyether ether ketone (PEEK), etc. ${ }^{[3]}$

\subsubsection{Metal materials for 3D printing}

3D printing metal materials mainly come in powder form and wire form. Powder materials are the most commonly used materials, which can be used in various 3D printing processes such as laser selective melting, laser near-net shaping, and electron beam selective melting; wire materials are suitable for arc additive manufacturing and other processes. According to the type of material, 3D printing metal materials can be divided into iron-based alloys, titanium and titanium-based alloys, nickel-based alloys, cobalt-chromium alloys, aluminum alloys, copper alloys, and precious metals.

\subsubsection{Ceramic materials for 3D printing}

Traditional ceramics can be defined as those ceramic products that make up the silicate industry, mainly including clay, cement and silicate glass. The raw materials of traditional ceramics are mostly natural mineral raw materials, which are widely distributed and low in price. They are suitable for the manufacture of daily ceramics, sanitary ceramics, refractory materials, abrasives, building materials, etc.

\subsection{Market situation of 3D printing materi- als}

\subsubsection{Type of 3D printing materials}

Metal consumables accounted for 39.4\%, lower than plastic $46.5 \%$, mainly because the industrialization of metal printing is in a rapid expansion stage, and the growth of equipment is ahead of the growth of material consumption. Non-metal 3D printing usually uses plastics, resin materials, etc., and metal 3D printing usually uses various alloy powders and wires.

\subsubsection{The material cost ratio continues to in- crease}

With the growth of molding accuracy, molding quality, and printing time, the proportion of equipment, maintenance and labor has gradually increased. In the SLM process with the best print quality, equipment, maintenance and labor costs account for the highest proportion, which also has patent protection Factors, but under the trend of increasing printing efficiency and scale effects. The proportion of material costs will also be further increased.

\subsection{Application field of 3D printing materials}

The main application areas of 3D printing include industrial equipment, consumer goods and electronics, automobiles, aerospace, and medical, accounting for more than $75 \%$. At present, the approximate composition ratio of $3 \mathrm{D}$ printing applications: industrial equipment accounts for $18 \%$, consumer products and electronics account for $17 \%$, automobiles account for $16 \%$, aerospace accounts for $15 \%$, medical/dental accounts for $13 \%$, scientific research modeling, military, construction, etc. Accounted for $22 \%$. It can be seen that $3 \mathrm{D}$ printing is not only applied to low-end manufacturing companies but also applied to high-end technology. It is a technology that conforms to the call of the times.

\subsection{Market size related to 3D printing}

The 3D printing market continues to exceed expectations. In the past 27 years, the compound growth rate of the global 3D printing industry has exceeded 27\%, and the market size in 2015 reached 5.165 billion US dollars. The domestic 3D printing market has doubled in succession over the past four years. In 2015, the size of the Chinese market was around RMB 7.8 billion.

The aerospace field is the fastest growing field of metal 3D printing. 3D printing is widely used in aircraft wing and fuselage, engine parts, aviation station parts replenishment, and drone systems. According to our calculations, the $3 \mathrm{D}$ printing aviation engine parts market will reach 1.25 trillion in the next 20 years ${ }^{[4]}$.

\section{Problems of 3D printing in the}




\section{Chinese market}

There are currently a large number of 3D printing technology in the Chinese market, but few can be called high-end technology industries. The reasons are roughly as follows: the selection of materials and the high cost. These problems make some small workshop-style manufacturing workshops unable to achieve sustainable production and low commercialization.

\subsection{Material selection}

\subsubsection{Engineering plastics}

It is currently one of the most widely used materials in the domestic market. It has good mechanical and physical properties and can be used to manufacture industrial parts or housings. Commonly used are nylon, polycarbonate, acrylonitrile-butadiene-styrene copolymer materials, etc. ABS is a commonly used molding material for FDM technology. It has good strength, toughness and impact resistance, and can be machined, electroplated and painted. There are many colors of this type of material, such as white, black, gray, red, blue, etc., so it has a wide range of applications in the automotive industry, home appliance industry, and consumer electronics.

However, in its development process, there are still certain problems, that is, sometimes, the printing materials do not fully meet the needs of the printed model, and sometimes, the 3D printing materials will have some color differences and defects. The emergence of these problems has hindered consumers' desire to consume printers and printing materials. Therefore, attention should be paid to these problems ${ }^{[5]}$.

\subsubsection{Metal materials}

The metal powders used in 3D printing generally require high purity, good sphericity, narrow particle size distribution, and low oxygen content. At present, the metal powder materials used in 3D printing mainly include titanium alloy, cobalt-chromium alloy, stainless steel and aluminum alloy materials, and there are also precious metal powder materials such as gold and silver used for printing jewelry.

Titanium is an important structural metal. Titanium alloys are widely used in the production of aircraft engine compressor parts, as well as various structural parts of rockets, missiles and airplanes due to their high strength, good corrosion resistance and high heat re- sistance. Cobalt-chromium alloy is a high-temperature alloy with cobalt and chromium as the main components. It has excellent corrosion resistance and mechanical properties. The parts made with it have high strength and high temperature resistance. The titanium alloy and cobalt-chromium alloy parts manufactured by 3D printing technology have very high strength and precise dimensions. The smallest size that can be produced can reach $1 \mathrm{~mm}$, and the mechanical properties of the parts are better than the forging process.

\subsection{Cost problem}

It is well known that in the initial stage of 3D printing, the cost of use is very expensive, and ordinary small and medium-sized enterprises can hardly bear the high cost in the initial stage of use. It is like designing a mold. Although his design cost is high, when the number of products increases, the cost can be reduced to a certain extent. The cost reduction is mainly optimized from these aspects.

Calculate how much material (cubic centimeters) is used by measuring the volume of the material. Ordinary products are cheaper to use fewer materials; ceramic products are cheaper to use a smaller surface area.

\subsubsection{Reduce the design scale}

Scale down the design and reduce the product volume. Reducing the design ratio is an efficient way to reduce printing costs.

\subsubsection{Hollow design}

3D printing can easily produce hollow effects, which is a very attractive feature of $3 \mathrm{D}$ printing. This will not only save costs economically, but also protect the environment and reduce material usage ${ }^{[6]}$.

\subsubsection{Hollow out design}

For materials other than ceramics, the hollow design on the surface is an effective way to reduce costs (reducing materials while increasing surface area). This method greatly affects the overall appearance of the product.

\subsection{Use crowd}

In recent years, 3D printing is not so much a high-tech, it is more a "toy". Because it is not just factories, there are 3D printing in many schools, processing plants, and even a small number of families in China. 
This is an upsurge of development, a stage of technological development for a new generation of development ${ }^{[7]}$.

\section{Future development potential}

With the background of Industry 4.0 and the strong support of governments of various countries, it is expected that the global 3D printing industry will still be in a period of rapid growth in the next ten years. According to IDC's forecast, the global 3D printing industry output value will reach 28.9 billion US dollars in 2020. As a strong promoter of the global 3D printing industry, China will invest more energy in 3D printing professional training, industry standard formulation, and cutting-edge technology research and development.

\section{Conclusion}

Looking forward to the future, the types of 3D printed products will be more and more diverse, ranging from airplanes and cars to toys and dentures. These products will bring people a brand-new audiovisual experience with exquisite design and relatively complete functions. In terms of personalized customization, 3D printing will also bring more surprises to people.

\section{References}

1. Hu T. Research on the application and development prospect of chemical materials in 3D printing industry (in Chinese). China Petroleum and Chemical Industry Standard and Quality 2019; 39(2): 188-189.

2. Zhang X, Tang S, Zhao H, et al. Research status and key technologies of 3D printing technology (in Chinese). Materials Engineering 2016; 44(2): 122-128.

3. Yang D, Xia X. 3D printing of biological materials: progress and clinical application (in Chinese). Chinese Journal of Tissue Engineering Research 2017; 21(18): 2927-2933.

4. Liu L, Xu W, Wang L, et al. Research progress of geometric calculation in 3D printing (in Chinese). Chinese Journal of Computers 2015; 38(6): 12431267.

5. Jia M, Chen K, Xue P. Research on high performance fiber reinforced thermoplastic composite molding technology and equipment (in Chinese). Beijing University of Chemical Technology and Tianjin University of Technology. Summary of the 2nd National Advanced Composite Materials Science and Application Symposium. Professional Committee of New Chemical Materials of China Chemical Society; Beijing University of Chemical Technology, Tianjin Polytechnic University, China Science Energy and Engineering Technology Research Institute (Beijing) Co. Ltd; 2018. p. 38.

6. Zhang L. Application and development of chemical materials in 3D printing field (in Chinese). Chemical Industry 2017; 35(2): 37-41.

7. Zhang S, Xu Y, Sun S, et al. Research and development status of 3D printing materials (in Chinese). China Plastics 2016; 30(1): 7-14. 